\title{
Energetically stable singular vortex cores in an atomic spin-1 Bose-Einstein condensate
}

\author{
Justin Lovegrove, Magnus O. Borgh, and Janne Ruostekoski \\ School of Mathematics, University of Southampton, Southampton, SO17 1BJ, United Kingdom
}

(Received 27 April 2012; published 12 July 2012)

\begin{abstract}
We analyze the structure and stability of singular singly quantized vortices in a rotating spin-1 Bose-Einstein condensate. We show that the singular vortex can be energetically stable in both the ferromagnetic and polar phases despite the existence of a lower-energy nonsingular coreless vortex in the ferromagnetic phase. The spin-1 system exhibits an energetic hierarchy of length scales resulting from different interaction strengths, and we find that the vortex cores deform to a larger size determined by the characteristic length scale of the spin-dependent interaction. We show that in the ferromagnetic phase the resulting stable core structure, despite apparent complexity, can be identified as a single polar core with an axially symmetric density profile which is nonvanishing everywhere. In the polar phase, the energetically favored core deformation leads to a splitting of a singly quantized vortex into a pair of half-quantum vortices that preserves the topology of the vortex outside the extended core region, but breaks the axial symmetry of the core. The resulting half-quantum vortices exhibit nonvanishing ferromagnetic cores.
\end{abstract}

DOI: 10.1103/PhysRevA.86.013613

PACS number(s): 03.75.Lm, 03.75.Mn, 67.85.Fg, 05.30.Jp

\section{INTRODUCTION}

In the textbook examples of superfluids, liquid ${ }^{4} \mathrm{He}[1]$ and Bose-Einstein condensates (BECs) [2] of spinless or spin-polarized atoms, quantized vortices occur as quantized circulation around an empty vortex core whose size is determined by a characteristic healing length. In a BEC of atoms whose spin degree of freedom is not frozen by magnetic fields [3], spin rotations and condensate phase combine to form a larger set of physically distinguishable degenerate states. This is analogous to liquid ${ }^{3} \mathrm{He}$ where superfluidity is formed by Cooper pairs of fermions that exhibit a nonzero spin and orbital angular momentum, resulting in a rich phenomenology of phases with different broken symmetries [4]. A variety of different vortex configurations [5] and other defects and textures [6] have been theoretically studied and experimentally observed in the resulting multicomponent order-parameter manifolds of superfluid liquid ${ }^{3} \mathrm{He}$. There are obvious parallels [6] to similar objects in cosmology [7] and quantum field theory [8].

Consequently, it is not surprising that in multi-component BECs, there has been an increasing interest in theoretical studies of topological defects and textures [9-39] as well as vector solitons [40-47]. The theoretical work has been produced in parallel with a rapid experimental progress on spinor BECs, e.g., in controlled preparation of coreless spinor vortices [48-50], in studies of spin-texture formation [51,52], and in nonequilibrium vortex production during rapid phase transitions [53].

An atomic spin-1 BEC exhibits two phases of the groundstate manifold, ferromagnetic (FM) and polar, with distinct broken symmetries. In the FM phase the ground state of a sufficiently rapidly rotating atom cloud is formed by nonsingular, coreless vortices in which the order parameter is well defined everywhere $[17,18,26,28,36]$. Similar coreless vortices were first described in superfluid liquid ${ }^{3} \mathrm{He}[54,55]$ and were recently experimentally phase imprinted on a spinor BEC [49]. Due to the topology of the FM ground-state manifold, which is defined by the group of spin rotations, it is also possible to form a singular vortex $[15,16]$, whose stability and structure, however, are much less well understood.

Here we show that singular, singly quantized vortices can be energetically stable in both the FM and polar phases of a spin-1 BEC. In the FM phase this is despite the fact that the coreless vortex has a lower energy. Although a singular vortex would also not be nucleated by rotation, once created, for example by phase imprinting, it can be stabilized in a rotating trap. In the polar phase, the singular vortex undergoes a core deformation to a pair of half-quantum vortices in an extended vortex-core region, where the broken order-parameter symmetry of the polar ground-state manifold is restored (see Fig. 1).

In a singular defect the singularity of the order parameter is contained by a defect core. Unlike in scalar superfluids, in the spinor BEC this does not imply that the density must vanish: it is also possible to accommodate the singularity by requiring the spinor wave function to be orthogonal to the ground-state manifold at the precise location of the singularity. The different possibilities for the defect-core structure lead to an energetic hierarchy of different characteristic length scales [23]: Depending on the ratio of the spin $\xi_{F}$ and density $\xi_{n}$ healing lengths associated with the two interaction strengths of a spin-1 $\mathrm{BEC}$, it can be energetically more favorable to force the orderparameter value to be orthogonal to the ground-state manifold at the defect singularity than to force the density to zero. This can lead to unexpected core structures. In Ref. [23] it was shown that in the polar phase of a spin-1 BEC a singular point defect with a vanishing density at the singularity can spontaneously deform to a ring defect with a FM core and a nonvanishing density. Recently symmetry classification using homotopy theory was used in the analysis of defect cores in Ref. [56].

In a spin-1 BEC the polar and FM phases differ by the local expectation value of the spin magnitude. The size of the filled vortex core is then determined by $\xi_{F}$, which defines the length scale over which the spin magnitude heals when locally perturbed. This is in general much larger than the size $\xi_{n}$ of a density-depleted vortex core. Large wave-function gradients close to the defect singularity result in a large orderparameter bending energy. Energetically, the system therefore 


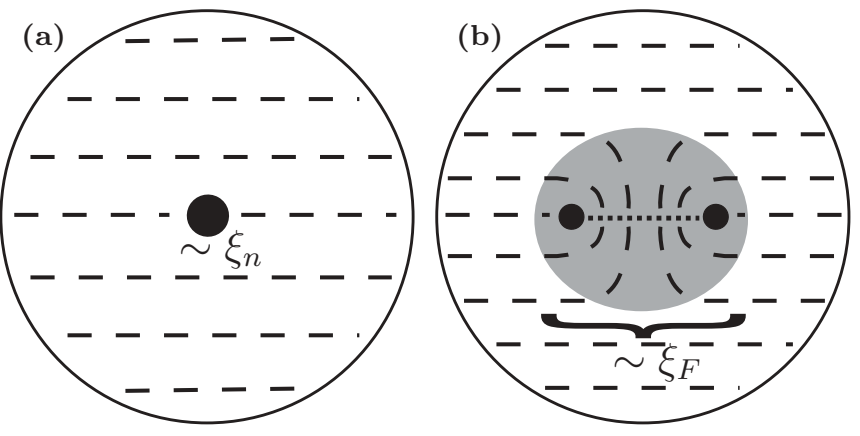

FIG. 1. Schematic illustration of two vortex-core structures with the same topology for a singly quantized singular vortex in the polar phase of a spin- 1 condensate. In (a) the atom density vanishes at the vortex-line singularity with the core size determined by the characteristic length scale $\xi_{n}$ (healing length) associated with the spin-independent interaction strength. In (b) the atom density is nonvanishing in the core region, whose size is determined by the characteristic length scale $\xi_{F}$ of the spin-dependent interaction strength. The vortex-line singularity has now split into two halfquantum vortices with the atoms in the ferromagnetic phase at the precise location of the singularities. In both panels we show the nematic axis as dashed lines and the dotted line in (b) indicates a disclination plane for the nematic axis. Inside the core region (shaded area) of (b) the broken symmetry of the polar ground-state manifold is restored (as explained in the text). Outside the core the topological properties of the vortex are the same as those in (a).

prefers the larger core size and a nonvanishing atom density with correspondingly lower bending energy. Outside the filled core region of size $\xi_{F}$, the topology of the vortex is the same as in the case of a zero-density vortex line. In that region the orderparameter bending energy is not sufficient to excite the system away from the ground-state manifold, and we find a welldefined broken order-parameter symmetry of either the polar or the FM phase. It is only inside the filled core of size $\xi_{F}$ that the vortex structure differs. Inside the core the order-parameter bending energy restores the order-parameter symmetry of the full spin-1 condensate wave function by exciting the system out of the ground-state manifold by mixing the polar and FM phases. In our numerical simulations this is indicated by a continuously varying spin magnitude across the vortex core.

We show that in the case of a singular singly quantized FM vortex the apparent complexity of the core can in this way be explained as the formation of a single core with $|\langle\hat{\mathbf{F}}\rangle|=0$ at the singularity: An initial singular vortex is formed by overlapping vortex lines in the spinor components. As the system relaxes, the density depletion is avoided by separating the vortex lines. By a rotation of the spinor basis, the axial symmetry of density profiles of the individual spinor components with perfectly overlapping vortex lines is explicitly restored, and the vortex is identified as one in which the spin vector winds by $2 \pi$ around a polar core. We find both axisymmetric and nonaxisymmetric solutions for the singular vortex in the FM phase, indicating close energetic degeneracy of the solutions.

In the polar phase, we use the same analysis to show how it is energetically favorable for the system to spontaneously break axial symmetry by splitting the core of a singly quantized vortex into two FM cores. The resulting spinor wave function shows a complex combination of vortex lines with highly deformed anisotropic cores. However, by transforming the spinor wave function to the basis specified by the direction of the spin in the FM cores, the spinor structure is identified as a pair of half-quantum vortices. Outside the deformed core, the topology of the initial singly quantized vortex is preserved. The splitting can be understood from the nematic symmetry properties of the polar order parameter. A stable nonaxisymmetric singular vortex with a nonzero superfluid density at the core has been theoretically predicted $[57,58]$ and experimentally observed [59] in superfluid liquid ${ }^{3} \mathrm{He}$.

Here we analyze the energetic stability of the singular vortices and explain the structures of their cores by numerical simulations in the framework of mean-field theory. The paper is organized as follows: In Sec. II we give a brief overview of mean-field theory for the spin-1 BEC and explain the general concepts used in our analysis. In Sec. III we demonstrate the energetic stability and explain the core structure of a singular vortex in the FM phase. Section IV applies a similar analysis to study energetic stability and identify the core structure of a singular vortex in the polar phase. We conclude with a brief summary of our findings in Sec. V. Analytic properties of the vortex solutions and the basis transformations are provided in the Appendix.

\section{SPIN-1 MEAN-FIELD THEORY}

In our analysis of singular vortices of a spin-1 atomic BEC, we consider the classical (Gross-Pitaevskii) mean-field theory of a harmonically trapped system that results in a spatially nonuniform atom density. In an optical trapping potential the atomic spin is not frozen by magnetic fields and the spin-1 $\mathrm{BEC}$ is represented by a normalized three-component spinor $\zeta(\mathbf{r})$ in the basis of spin projection onto the $z$ axis. Together with the density $n(\mathbf{r})=|\Psi(\mathbf{r})|^{2}$, this specifies the macroscopic condensate wave function

$$
\Psi(\mathbf{r})=\sqrt{n(\mathbf{r})} \zeta(\mathbf{r})=\sqrt{n(\mathbf{r})}\left(\begin{array}{c}
\zeta_{+}(\mathbf{r}) \\
\zeta_{0}(\mathbf{r}) \\
\zeta_{-}(\mathbf{r})
\end{array}\right), \quad \zeta^{\dagger} \zeta=1 .
$$

The Hamiltonian density in the frame rotating with frequency $\Omega$ around the $z$ axis is $[2,9,60]$

$$
\begin{aligned}
\mathcal{H}= & \frac{\hbar^{2}}{2 m}|\nabla \Psi|^{2}+V(\mathbf{r}) n+\frac{c_{0}}{2} n^{2}+\frac{c_{2}}{2} n^{2}|\langle\hat{\mathbf{F}}\rangle|^{2} \\
& +g_{1} n\langle\mathbf{B} \cdot \hat{\mathbf{F}}\rangle+g_{2} n\left\langle(\mathbf{B} \cdot \hat{\mathbf{F}})^{2}\right\rangle-\Omega\left\langle\hat{L}_{z}\right\rangle,
\end{aligned}
$$

where $V(\mathbf{r})$ is an external trapping potential, $m$ is the atomic mass, and $\left\langle\hat{L}_{z}\right\rangle=-i \hbar \Psi^{\dagger}\left(x \partial_{y}-y \partial_{x}\right) \Psi$ denotes the $z$ component of the angular momentum operator. The spin operator $\hat{\mathbf{F}}$, whose expectation value $\langle\hat{\mathbf{F}}\rangle=\zeta_{\alpha}^{\dagger} \hat{\mathbf{F}}_{\alpha \beta} \zeta_{\beta}$ yields the local spin vector, is given by a vector of spin-1 Pauli matrices. The first two terms in the second line of Eq. (2) describe linear and quadratic Zeeman shifts, respectively, in the presence of a weak external magnetic field $\mathbf{B}$. In this paper we assume an axially symmetric harmonic confinement such that

$$
V(\mathbf{r})=\frac{1}{2} m\left[\omega_{\perp}^{2}\left(x^{2}+y^{2}\right)+\omega_{z}^{2} z^{2}\right],
$$

from which we also define the transverse oscillator length $l_{\perp}=\sqrt{\hbar / m \omega_{\perp}}$.

The spins of two colliding spin-1 atoms may combine to a relative angular momentum of either 0 or 2 . This implies that 
the contact interaction results from two different contributions, corresponding to the two scattering channels with different $s$-wave scattering lengths $a_{0}$ and $a_{2}$. The two scattering contributions lead to the two interaction terms in Eq. (2). The strength of the interactions may be calculated using angular momentum algebra, and we have $c_{0}=4 \pi \hbar^{2}\left(2 a_{2}+a_{0}\right) / 3 m$ and $c_{2}=4 \pi \hbar^{2}\left(a_{2}-a_{0}\right) / 3 m$ [2]. In addition the Hamiltonian density (2) may include magnetic dipole-dipole interaction terms that can influence the spin textures [51,61].

The spin-dependent interaction term $c_{2}$ in Eq. (2) determines the spin magnitude in a uniform ground-state spin distribution. If $c_{2}<0$, as is the case for ${ }^{87} \mathrm{Rb}$, the spindependent contribution to the interaction energy will favor the FM state with $|\langle\hat{\mathbf{F}}\rangle|=1$ throughout the BEC. Conversely if $c_{2}>0$, as for ${ }^{23} \mathrm{Na}$, the polar state with $|\langle\hat{\mathbf{F}}\rangle|=0$ will be favored.

The two interaction strengths $c_{0}$ and $c_{2}$ are each associated with a characteristic length scale. From the spin-independent interaction we can derive the healing length $\xi_{n}=\left(8 \pi c_{0} n\right)^{-1 / 2}$ that defines the length scale over which the density heals around a local depletion of the atom density [2]. This phenomenon is similar in a scalar BEC, which exhibits a healing length depending on the atom density and the scattering length. Due to the spin-dependent interaction term, we now, however, have an additional healing length, analogously given by $\xi_{F}=\left(8 \pi\left|c_{2}\right| n\right)^{-1 / 2}$. This defines the length scale over which the spin magnitude $|\langle\hat{\mathbf{F}}\rangle|$ heals when locally perturbed.

As in a scalar BEC, single-valuedness of the order parameter may be maintained at a defect singularity by requiring that the density vanishes there. The size of the defect core is then given by the healing length $\xi_{n}$. However, the spinor order parameter makes it possible to maintain a nonzero density at the cost of requiring that the wave function at the singularity become orthogonal to the ground-state manifold. For example, a singularity in the FM manifold where $|\langle\hat{\mathbf{F}}\rangle|=1$ can be accommodated by having $|\langle\hat{\mathbf{F}}\rangle|=0$ on the vortex line. This constitutes a local perturbation of the spin magnitude, and so its length scale is determined by $\xi_{F}$, which is usually larger than $\xi_{n}$. The energetic cost of the local change in spin magnitude due to increased interaction energy may be offset by the lower bending energy in the larger core.

The two phases of the spin-1 BEC are described by very different order parameters, which leads to dramatically different possible vortex states. In the following we shall first consider the FM phase; we show that a singular, singly quantized vortex can be energetically stable, and describe how its core structure can be understood in terms of the energetics of characteristic length scales (Sec. III). We will then apply a similar analysis to show how the deformed core of a stable singly quantized vortex in the polar phase can be identified as a pair of half-quantum vortices (Sec. IV).

\section{STABILITY AND CORE DEFORMATION OF A SINGULAR FERROMAGNETIC VORTEX}

We first consider vortices in the FM phase of a spin1 BEC. The system becomes FM when the interaction term $c_{2}<0$ in the Hamiltonian (2); energetically it is then favorable to maximize the spin magnitude everywhere in space, so that $|\langle\hat{\mathbf{F}}\rangle|=1$. A general FM spinor wave function can be constructed from the representative spinor $\zeta=(1,0,0)^{T}$ with $\langle\hat{\mathbf{F}}\rangle=\hat{\mathbf{z}}$ by incorporating a macroscopic condensate phase $\phi$ and by a spin rotation $U(\alpha, \beta, \gamma)=$ $\exp \left(-i F_{z} \alpha\right) \exp \left(-i F_{y} \beta\right) \exp \left(-i F_{z} \gamma\right)$, defined by three Euler angles. We obtain

$$
\begin{aligned}
\zeta^{\mathrm{f}} & =e^{i \phi} U(\alpha, \beta, \gamma)\left(\begin{array}{l}
1 \\
0 \\
0
\end{array}\right) \\
& =\frac{e^{-i \gamma^{\prime}}}{\sqrt{2}}\left(\begin{array}{c}
\sqrt{2} e^{-i \alpha} \cos ^{2} \frac{\beta}{2} \\
\sin \beta \\
\sqrt{2} e^{i \alpha} \sin ^{2} \frac{\beta}{2}
\end{array}\right),
\end{aligned}
$$

where $\gamma^{\prime}=\gamma-\phi$. The local spin vector is then given by $\langle\hat{\mathbf{F}}\rangle=(\cos \alpha \sin \beta, \sin \alpha \sin \beta, \cos \beta)$.

Order-parameter space is the manifold of energetically degenerate spinors $\zeta$. Degenerate FM spinors [Eq. (4)] differ only by rotations in spin space given by the Euler angles $\alpha$, $\beta$, and $\gamma^{\prime}$. The order-parameter space therefore corresponds to the group of three-dimensional rotations $\mathrm{SO}(3)$.

The topological stability of line defects is characterized by the way closed contours encircling the defect map into orderparameter space [62]. If the order-parameter space image of such a closed loop can be contracted to a point, the defect is not topologically stable. $\mathrm{SO}(3)$ may be represented geometrically as $S^{3}$ (the unit sphere in four dimensions) with diametrically opposite points identified. The only closed loops that cannot be contracted to a point are those connecting such identified points an odd number of times (but these loops can all be deformed into one another). There are therefore only two distinct classes of vortices: singular vortices corresponding to noncontractible loops, and nonsingular vortices corresponding to contractible loops $[9,60]$. All the singular vortices with an odd-integer winding number are therefore topologically equivalent to a singly quantized singular vortex and all singular vortices with an even-integer winding number are topologically equivalent to a nonsingular vortex-free state. Mathematically, this is indicated by the first homotopy group of $\mathrm{SO}(3)$, which has two elements $\left[\pi_{1}(\operatorname{SO}(3))=\mathbb{Z}_{2}\right]$ that represent the two topological equivalence classes for the vortices. Typical examples of a nonsingular coreless vortex forming a continuous spin texture and a singular spin vortex with radial and cross disgyrations of the spin vector are schematically illustrated in Fig. 2.

In the FM phase, circulation need not be quantized [9]. A striking manifestation of this fact is the possibility of having a coreless vortex, which may be written

$$
\zeta^{\mathrm{cl}}=\frac{1}{\sqrt{2}}\left(\begin{array}{c}
\sqrt{2} \cos ^{2} \frac{\beta}{2} \\
e^{i \varphi} \sin \beta \\
\sqrt{2} e^{2 i \varphi} \sin ^{2} \frac{\beta}{2}
\end{array}\right),
$$

where $\varphi$ is the azimuthal angle and the Euler angle $\beta$ varies with the radial distance $\rho=\sqrt{x^{2}+y^{2}}$ from the $z$ axis. The spin texture is kept continuous by having $\beta \rightarrow 0$ as $\rho \rightarrow$ 0 . The coreless vortex carries angular momentum, yet can be continuously deformed into a vortex-free state. Because the FM order parameter is well defined and nonsingular everywhere in space (a coreless vortex forms a continuous spin 


$$
\text { (a) }
$$
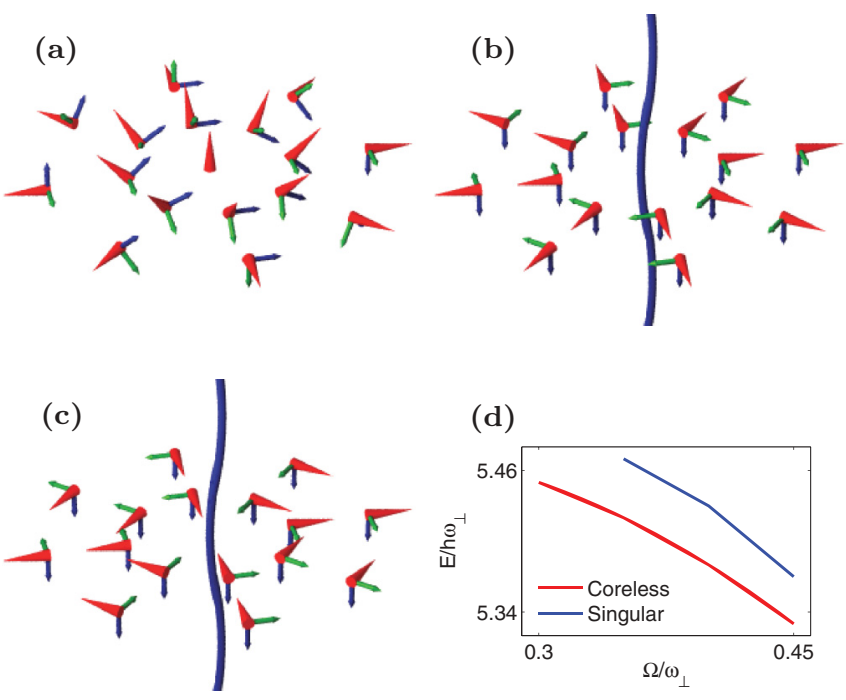

FIG. 2. (Color online) Schematic illustrations of FM vortex states. (a) The nonsingular, coreless vortex is formed as a combined disgyration of the spin vector (cones) and a spin rotation about the local spin vector (indicated by the orthogonal vectors). The vortex is nonsingular and the spin texture is continuous. (b) The singular FM spin vortex is formed as a radially oriented disgyration of the spin vector around the singular core, which is filled by the polar phase. (c) The class of singular vortices contains several different spin configurations, for example the cross-disgyration shown. They can all be deformed to the singular spin vortex through local spin rotations. (d) Energies of the stable coreless (lower line) and singular vortices as functions of the frequency of rotation in an isotropic trap using $N c_{0}=1000 \hbar \omega_{\perp} l_{\perp}^{3}$ and $N c_{2}=-320 \hbar \omega_{\perp} l_{\perp}^{3}$. The coreless vortex is lower in energy in the whole frequency range.

texture) the coreless vortex belongs to the class of nonsingular vortices. It is the spinor-BEC analog of the Anderson-Toulouse and Mermin-Ho vortices in ${ }^{3} \mathrm{He}$ [54,55], which differ by the imposed boundary conditions at the container wall. The superfluid velocity [9]

$$
\mathbf{v}=\frac{\hbar}{m \rho}(1-\cos \beta) \hat{\boldsymbol{\varphi}}
$$

goes smoothly to zero at the center of the vortex but increases away from it as $\beta$ increases. The spin forms a characteristic fountain texture. In the atomic gas, there is no hard container wall, and the amount by which $\langle\hat{\mathbf{F}}\rangle$ turns as $\rho$ increases from the vortex center to the edge of the cloud is not fixed. The total circulation can thus vary smoothly as the value of $\beta$ at the edge of the cloud adapts to the imposed rotation [63].

The simplest way to construct a singly quantized singular vortex in the FM phase is as a $2 \pi$ winding of the condensate phase $\phi$. The vortex can then be described by the spinor

$$
\zeta^{\mathrm{s}}=\frac{e^{i \varphi}}{\sqrt{2}}\left(\begin{array}{c}
\sqrt{2} \cos ^{2} \frac{\beta}{2} \\
\sin \beta \\
\sqrt{2} \sin ^{2} \frac{\beta}{2}
\end{array}\right),
$$

where the density is required to vanish on the singular vortex line along the $z$ axis (where all three spinor components are singular). The Euler angle $\beta$ is arbitrary but constant, giving a uniform spin distribution (which, without loss of generality, we assume to be in the $x z$ plane such that $\alpha=0$ in Eq. (4)).

We may continuously deform $\zeta^{\mathrm{s}}$ into another vortex in the same class of topological line defects through purely local operations. For example we may rotate the spins into the radial disgyration ( $2 \pi$ rotation) of the spin vector shown in Fig. 2(b). This spin structure is derived from Eq. (4) by letting $\alpha=\varphi$ while $\gamma^{\prime}=0$,

$$
\zeta^{\mathrm{sv}}=\frac{1}{\sqrt{2}}\left(\begin{array}{c}
\sqrt{2} e^{-i \varphi} \cos ^{2} \frac{\beta}{2} \\
\sin \beta \\
\sqrt{2} e^{i \varphi} \sin ^{2} \frac{\beta}{2}
\end{array}\right)
$$

yielding a singular vortex with a circulation of the spin around the core (spin vortex). This is similar to a radial disgyration of the angular momentum in an analogous vortex structure in ${ }^{3} \mathrm{He}$ [4]. The FM order parameter is still singular at $\rho=0$ because a singularity is introduced in the FM spin vector. At $\beta=\pi$ we recover the vortex in Eq. (7) with only one spinor component occupied. Further local rotations of the spin allow us to construct additional members of the family of singular FM vortices. If we locally rotate all spins through $\pi / 2$ around the $z$ axis in Fig. 2(b) or Eq. (8), we change from the radial to a tangential disgyration, where $\langle\hat{\mathbf{F}}\rangle=\hat{\boldsymbol{\varphi}}$. A spin vortex could also be constructed from Eq. (4) by choosing $\alpha=-\varphi$. The radial disgyration is then replaced by the cross-disgyration illustrated in Fig 2(c). Because the $\mathrm{SO}(3)$ order-parameter manifold allows only two topologically distinct classes of vortices, all singly quantized, singular vortices can be transformed into each other by local spin rotations and this family of vortices is indeed quite large.

In order to determine the energetic stability of the vortex configurations and stable vortex core structures, we numerically minimize the energy of specific vortex states belonging to distinct topological equivalence classes. The energy relaxation is done by numerically propagating a coupled set of GrossPitaevskii equations derived from Eq. (2) in imaginary time using a split-step algorithm [64]. We consider an isotropic trap in a rotating frame with the nonlinearities $N c_{0}=1000 \hbar \omega_{\perp} l_{\perp}^{3}$ and $-640 \hbar \omega_{\perp} l_{\perp}^{3} \leqslant N c_{2} \leqslant-10 \hbar \omega_{\perp} l_{\perp}^{3}$, where $N$ is the total number of atoms. As an initial state for a singular singly quantized vortex we take the vortex of Eq. (7) in which case each spinor component exhibits a singly quantized vortex line. These all perfectly overlap with a vanishing density at the core. We also perform an energy minimization of the coreless, nonsingular vortex of Eq. (5). Coreless vortices have been shown to exist in the ground state of sufficiently rapidly rotating FM spin-1 BECs [17,18,26,28], and increasing the rotation rate of a vortex-free cloud is predicted to result in nucleation of coreless vortices in the system.

We find a single coreless vortex to be energetically stable in a sufficiently rapidly rotating trap, as shown in the stability diagram of Fig. 3(a). Figure 4(a) shows the characteristic fountainlike spin texture of the stable vortex. At slow rotation speeds the vortex exits the atom cloud and at faster rotation rates we observe nucleation of additional coreless vortices to the system. The threshold rotation frequency is increased at stronger nonlinearities. Our findings are consistent with those in Ref. [18].

For the singular initial-state vortex the corresponding stability diagram is displayed in Fig. 3(b). Although its core 
(a)

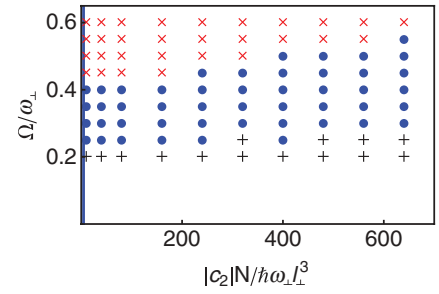

(b)

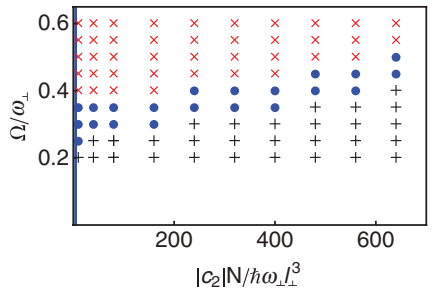

FIG. 3. (Color online) Energetic stability of the coreless (a) and the core-deformed singular vortex (b) in an isotropic trap for varying spin-dependent interaction strength $c_{2}<0$. The spin-independent interaction is fixed at $N c_{0}=1000 \hbar \omega_{\perp} l_{\perp}^{3}$. Blue dots $(\bullet)$ indicate that the vortex is energetically stable. A black plus $(+)$ indicates where the initial vortex leaves the cloud, whereas red crosses $(x)$ mark where additional vortices nucleate due to rotation. A blue (black) vertical line marks $c_{0} / c_{2} \simeq-216$ relevant for ${ }^{87} \mathrm{Rb}$ [65]. Note that with the parameters used here, this yields $N\left|c_{2}\right|=4.6 \hbar \omega_{\perp} l_{\perp}^{3}$. The line thus falls very nearly on top of the vertical axis and the two cannot be distinguished in the figure.

structure is deformed during energy relaxation (as we will discuss below), we find that the singular vortex is energetically stable for a range of rotation frequencies at all investigated values of $c_{2}$. This energetic stability of the singular vortex seems surprising since there also exists a stable coreless vortex with lower energy at the same rotation frequencies and nonlinearities. Our numerics also show that coreless vortices will nucleate due to rotation, whereas singular vortices will not. A comparison between the numerically calculated energies of a stable coreless vortex and a stable singular vortex as a function of the rotation frequency in an isotropic trap and with $N c_{0}=$ $1000 \hbar \omega_{\perp} l_{\perp}^{3}, N c_{2}=-320 \hbar \omega_{\perp} l_{\perp}^{3}$ is shown in Fig. 2(d). This may be contrasted with the vortex energetics of coreless and singular vortices in superfluid liquid ${ }^{3} \mathrm{He}-A$, where the singular vortex has lower energy, but the energy barrier for nucleation of the singular core is higher than that for forming a coreless vortex [66]. Singular vortices can be created by cooling a rotating normal fluid through the superfluid transition.

The coreless and the singular vortices belong to distinct topological equivalence classes and they cannot be continuously deformed into each other. For the singular vortex to
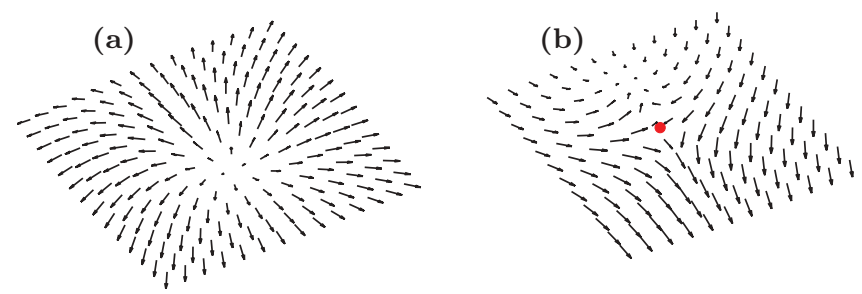

FIG. 4. (Color online) Numerically calculated spin textures in the stable FM vortex states in a rotating trap. The spin vector is shown in a cut perpendicular to the $z$ axis (the axis of rotation). (a) The spin vector in the coreless vortex exhibits a characteristic fountainlike structure and maintains $|\langle\hat{\mathbf{F}}\rangle|=1$ everywhere. (b) In the relaxed singular vortex, the spin vector winds by $2 \pi$ around the $x$ axis on a path encircling the singular vortex core (indicated by the dot), in which $|\langle\hat{\mathbf{F}}\rangle| \rightarrow 0$. This texture can be continuously deformed into that shown in Fig. 2(b). decay, the rotation frequency has to be sufficiently slow so that the vortex can exit the atom cloud and be replaced by a nucleating coreless vortex that enters from the edge of the cloud. We find a range of frequencies and nonlinearities [Fig. 3(b)] for which the singular vortex remains in the atom cloud and no additional coreless vortices nucleate. A single, singly quantized singular vortex thus represents a local minimum of the energy, topologically protected against decay to the lower-energy coreless vortex.

After demonstrating that the singular singly quantized vortex of Eq. (7) is energetically stable, we next study its vortex-core structure after the energy relaxation. The resulting vortex configuration with a stable vortex core is shown in Fig. 5(a). The vortex lines in the different spinor wave-function components have moved apart and no longer spatially overlap. We show in Fig. 6(a) a one-dimensional (1D) density cut along which the spatially separated vortices are aligned. The vortex line of the $\zeta_{0}$ component is located at the center of the trap and the vortices of the $\zeta_{ \pm}$components are symmetrically displaced from the center. This split-core solution appears to break the explicit axial symmetry of the spinor-component densities in Eq. (7). A similar core splitting has previously been demonstrated in 2D numerical simulations in Ref. [16]. We will show below how it is beneficial to analyze the vortex core using a spinor-basis transformation. In particular, after an appropriate transformation we can easily identify the location of the vortex, the nonvanishing atom density at the vortex-line singularity, and the axially symmetric density profiles of the spinor components in the new basis representation. In the vortex configuration displayed in Fig. 5(a) we may then identify the split-core vortex as spin winding around a core of nonvanishing atom density.

In order to analyze the vortex configuration of Fig. 5(a) we perform a basis transformation for the spinor wave function.
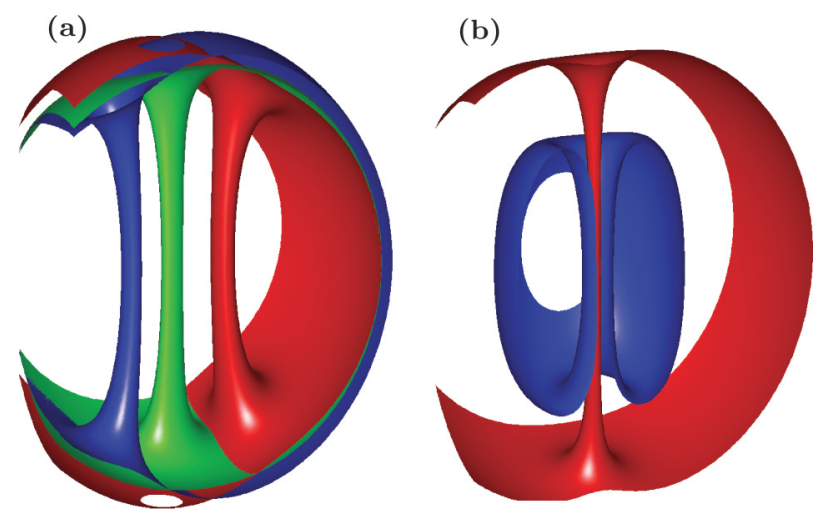

FIG. 5. (Color online) Split core of the singular FM vortex and restoration of a single core with explicit axial symmetry of the spinorcomponent densities illustrated by isosurfaces of the spinor wavefunction components $n\left|\zeta_{+}\right|^{2}$ [red (medium gray)], $n\left|\zeta_{0}\right|^{2}$ [green (light gray)], and $n\left|\zeta_{-}\right|^{2}$ [blue (dark gray)]. (a) In the spinor basis along the $z$ axis, the vortex lines in $\zeta_{+}^{(z)}, \zeta_{0}^{(z)}$, and $\zeta_{-}^{(z)}$ separate and the atom density is nonzero everywhere. (b) The axial symmetry of the density in each spinor component is restored by transforming the spinor to the basis of spin projection onto the $x$ axis. Vortex lines with opposite circulation in $\zeta_{ \pm}^{(x)}$ overlap. $\zeta_{0}^{(x)}$ (not shown) does not exhibit any vortex line [cf. Fig. 6(b)]. See also the Appendix for a qualitative analytic discussion of the relation between $\zeta^{(x)}$ and $\zeta^{(z)}$. 

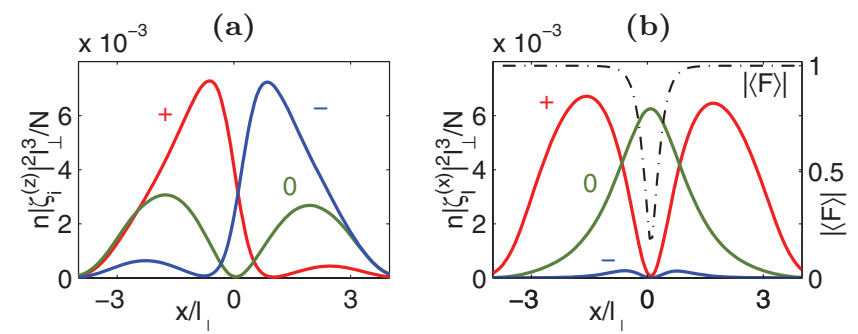

FIG. 6. (Color online) (a) Densities in the three spinor components $\zeta_{+}^{(z)}$ (red line marked by + ), $\zeta_{0}^{(z)}$ (green line marked by 0 ), and $\zeta_{-}^{(z)}$ (blue line marked by - ) on the axis connecting the vortex lines in the spinor components [cf. Fig. 5(a)]. (b) Densities in $\zeta_{+}^{(x)}$ (red line marked by +$), \zeta_{0}^{(x)}$ (green line marked by 0 ), and $\zeta_{-}^{(x)}$ (blue line marked by - ) on the same spatial axis after spinor-basis transformation. $|\langle\hat{\mathbf{F}}\rangle|$ (black dash-dotted line) goes to zero in the vortex core (the apparent nonzero minimum is due to the finite numerical resolution) which is filled by $\zeta_{0}^{(x)}$, keeping the density nonzero everywhere.

We transform the split-core spinor to the basis where spin is quantized along the $x$ axis as $\zeta^{(x)}=U^{-1}(0, \pi / 2,0) \zeta^{(z)}$, explicitly indicating the spinor basis by superscripts. In $\zeta^{(x)}$ the vortex appears as an opposite winding of the phase in the two components $\zeta_{ \pm}^{(x)}$. These vortex lines again overlap as shown in Figs. 5(b) and 6(b). Crucially, there is no vortex line in $\zeta_{0}^{(x)}$, and this component therefore fills the vortex cores of the two other components so that the density is nonvanishing everywhere. The single vortex core, which is readily apparent from Fig. 8, is thus explicitly restored in $\zeta^{(x)}$ by the transformation to the "natural basis" of the vortex. We identify the spinor wave function resulting from the basis transformation now as having the same structure as the singular vortex, defined in Eq. (8). In the Appendix we show how the core deformation and the relation between $\zeta^{(x)}$ and $\zeta^{(z)}$ can be understood qualitatively through an analytic treatment.

The spin structure of the stable vortex is shown in Fig. 4(b). The vortex line is oriented along the $z$ direction-the axis about which the trap is rotating. However, the spinor takes the form of Eq. (8) in the basis defined along the (corotating) $x$ axis. This vortex is singular, preserving the topology, and can be reached from Eq. (7) by local spin rotations, and could similarly be continuously transformed into the singular spin vortex [Fig. 2(b)]. The stable vortex core Fig. 4(b) has a broken spatial parity (the spin profile has an antisymmetric spatial parity close to the vortex core). This spin profile is nonaxisymmetric. We also find a stable axisymmetric vortex core. This is achieved by starting energy relaxation from Eq. (8), such that the radial disgyration of the spin vector is present already in the initial state. The spinor components and the resulting spin profile are shown in Fig. 7. The dependence of the final configuration on the initial state indicates a close energetic degeneracy of the two solutions.

To understand the vortex-core deformation it is also beneficial to compare the initial-state singular vortex of Eq. (7) to the vortex obtained in the energy minimization. In Eq. (7) each spinor component exhibits a singly quantized vortex. These overlapping vortex lines imply that the total density $n(\mathbf{r})$ must be zero on the singular line in order to maintain single-valuedness of the order parameter. The size of the vortex core is determined by the healing length $\xi_{n}$. The density (a)

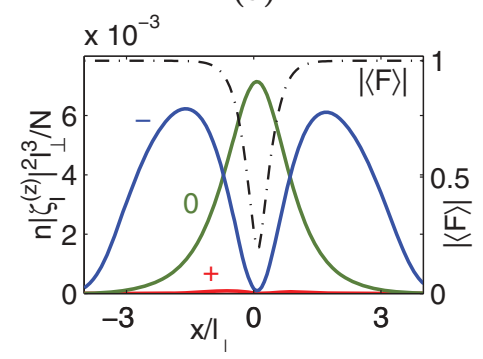

(b)

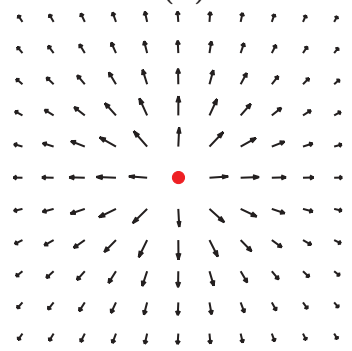

FIG. 7. (Color online) Axially symmetric spin vortex. (a) Densities of the spinor components together with the spin magnitude along a radial cut [lines and labels as in Fig. 6(a)]. The vortex lines in $\zeta_{ \pm}$ overlap perfectly at the position of the vortex core. (b) Relaxed spin profile in the $x y$ plane, showing the characteristic radial disgyration of the spin vector around the singular core. At large radii the spin vector bends out of the $x y$ plane. The vortex-line singularity is marked by a dot at the center.

depletion can be avoided by splitting the vortex core such that the vortex lines in the spinor components no longer overlap. Since the total condensate density then does not vanish at the vortex line where the order parameter is singular, we must now require that the spinor wave function become orthogonal to the ground-state manifold at the vortex singularity. In the FM manifold $|\langle\hat{\mathbf{F}}\rangle|=1$, so at the vortex line we must have $|\langle\hat{\mathbf{F}}\rangle|=0$, which represents the spin magnitude of the polar phase.

The spin magnitude of the numerically calculated singular vortex core is displayed in Figs. 6(b) and 8. We find that the value of the spin magnitude indeed rapidly approaches zero close to the vortex-line singularity (the small deviations from zero are due to the spatial resolution of the numerics). This indicates the formation of a polar vortex core, constituting a local violation of the spin condition for the ground-state FM manifold. An analytic description of the vortex solution is provided in the Appendix. The size of the vortex core is determined by the spin healing length $\xi_{F}$. The splitting is then

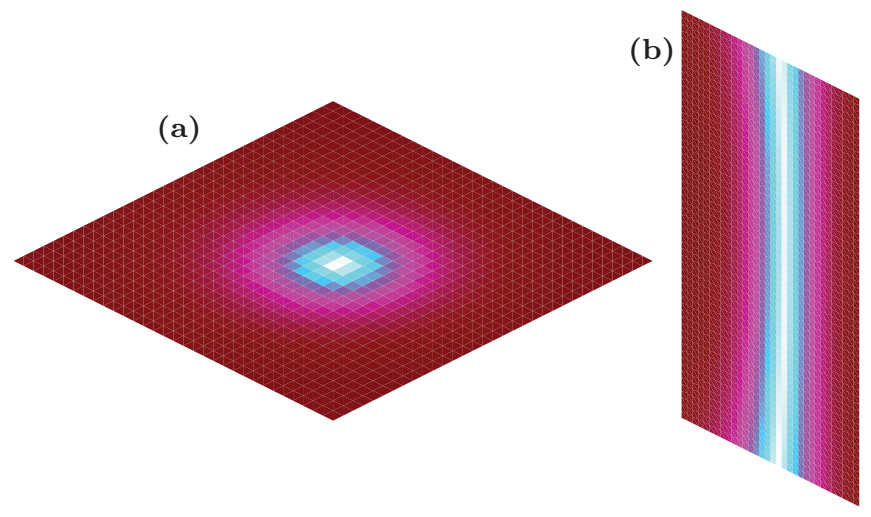

FIG. 8. (Color online) Spin magnitude $|\langle\hat{\mathbf{F}}\rangle|$, showing the core of the stable singular vortex of Fig. 6 in the FM phase, shown in the $x y$ plane (a) and $y z$ plane (b). Outside the vortex core, $|\langle\hat{\mathbf{F}}\rangle|=1$ [dark red (dark gray)] in the FM order-parameter manifold. In the core, the singularity is accommodated by enforcing $|\langle\hat{\mathbf{F}}\rangle|=0$ (white) while maintaining nonzero density. The size of the core region is determined by the spin healing length $\xi_{F}$. 
energetically favorable when $\xi_{F}$ allows a larger core size (i.e., when $\left.\xi_{F} \gtrsim \xi_{n}\right)$ such that the energy cost of violating $|\langle\hat{\mathbf{F}}\rangle|=1$ is smaller than that of depleting the density.

We find that the region where the spin magnitude deviates from $|\langle\hat{\mathbf{F}}\rangle|=1$ extends over the entire core size, determined by the spin healing length $\xi_{F}$. Outside the core region of the vortex the symmetry of the spin-1 BEC is broken according to the FM energy condition of the spin-dependent interaction energy, so that we have $|\langle\hat{\mathbf{F}}\rangle|=1$. Close to the singular vortex, however, the order-parameter bending energy restores the symmetry of the full spin-1 BEC wave function $\left(S^{5}\right.$ determined by a normalized spinor wave function of three complex components), mixing the FM and polar phases. The bending energy is enhanced very close to the vortex singularity due to the large density-gradient contributions that excite the system from the FM ground-state manifold. An analogous core deformation was previously found for a singular point defect in a polar spin-1 BEC in Ref. [23]. In that case an isotropic point defect with a vanishing density deformed to a ring defect with a FM core. This effect is closely related to the deformation of the core of a singular vortex in the polar phase described in Sec. IV.

In experiments a stable singular vortex could be prepared in a controlled way by phase-imprinting the initial singular vortex state of Eq. (7) in a rotating trap, so that the parameter values of the system belong to the stable region of the stability diagram displayed in Fig. 3(b). The initial-state vortex [Eq. (7)] is composed of perfectly overlapping singly quantized vortices in each of the spinor components. These could be phase imprinted using previously realized experimental techniques [67-69]. The stability diagram also indicates the conditions under which a singular vortex created in a phase transition [53] could potentially be stabilized.

In the above analysis, we have allowed the magnetization $M_{z}=N_{+}-N_{-}$, where $N_{ \pm}$are the total populations of $\zeta_{ \pm}$, to vary during the relaxation process. This in principle allows a spontaneous magnetization to develop in the system. In experiments, dissipative relaxation of energy due to atomic collisions may frequently conserve the magnetization. We have therefore also performed calculations where a weak magnetization is enforced throughout the energy-minimization procedure. We find the relaxed vortex configurations of Figs. 5-8, however, qualitatively similar to the ones where no fixed magnetization was forced.

Thus far we have considered an isotropic trap. We find that the results are qualitatively similar in an oblate trap with $\omega_{\perp} / \omega_{z}=0.1$. We find that also in this regime the singular vortex represents a local energetic minimum and is stable for a range of $\Omega$, again despite the fact that a lower-energy coreless-vortex solution exists. The parameter regions allowing stable nonsingular coreless and singular split-core vortices in the oblate trap are shown in Fig. 9. In the oblate trap case the simulations with fixed magnetization produce more symmetric vortex configurations than in the simulations where the magnetization varies freely but the qualitative features are the same in the two cases.

Application of a weak external magnetic field introduces a Zeeman shift between the spinor components according to Eq. (2). The spinor nature of the BEC is retained as long as the applied field is not too strong, $g_{1}|\mathbf{B}|, g_{2}|\mathbf{B}|^{2} \lesssim \mu$, where $\mu$ denotes the chemical potential. In the case of a small linear
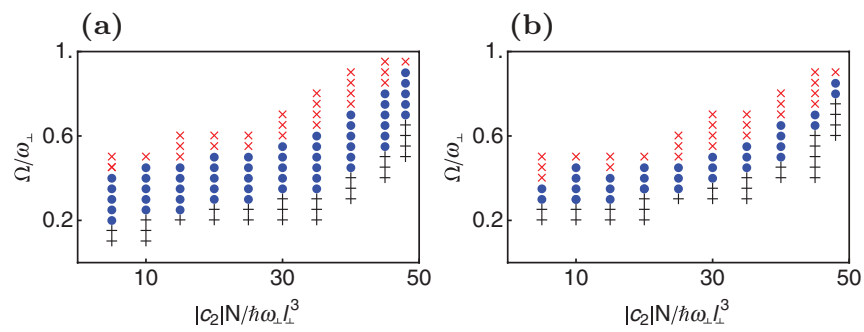

FIG. 9. (Color online) Stability of the FM coreless (a) and singular (b) vortices in a highly oblate trap, $\omega_{\perp} / \omega_{z}=0.1$, with $N c_{0}=50 \hbar \omega_{\perp} l_{\perp}^{3}$ and $-48 \hbar \omega_{\perp} l_{\perp}^{3} \leqslant N c_{2} \leqslant-5 \hbar \omega_{\perp} l_{\perp}^{3}$. (Symbols as in Fig. 3.)

Zeeman splitting $g_{1}|\mathbf{B}|$ (taking $\mathbf{B}$ along the $z$ axis) we find that the coreless and singular vortices are both stable, with the coreless vortex lower in energy. The Zeeman splitting will tend to align the spins with the applied field. This causes the energy of the coreless vortex to increase as maintaining the fountainlike spin structure becomes energetically less favorable. Thus we find that for $g_{1}|\mathbf{B}| \gtrsim 0.2 \hbar \omega_{\perp}$ the coreless vortex is no longer stable. The singular vortex, on the other hand, remains energetically stable for all $g_{1}|\mathbf{B}|$ considered (up to $0.8 \hbar \omega_{\perp}$ ), as shown in Figs. 10(a) and 10(b). For a sufficiently large linear Zeeman splitting, the ideal spinor basis to analyze the singular vortex core becomes the one defined by the magnetic field.

A quadratic Zeeman splitting, on the other hand, does not destroy the stability of the coreless vortex, but for $g_{2}|\mathbf{B}|^{2} \lesssim$ $-0.1 \hbar \omega_{\perp}$ the singular vortex is no longer energetically stable (a)

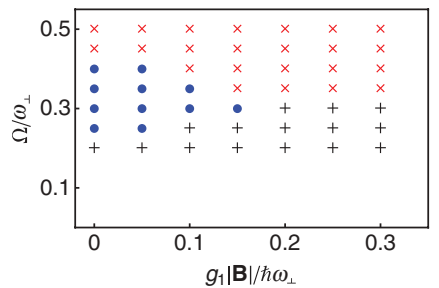

(c)

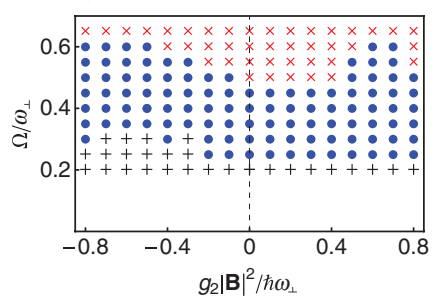

(b)

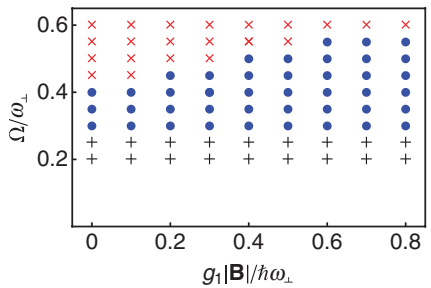

(d)

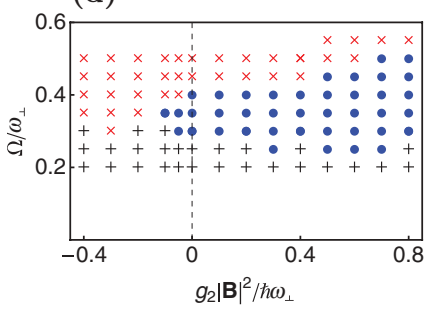

FIG. 10. (Color online) Effects of linear and quadratic Zeeman splitting on the stability of the FM vortices in an oblate trap $\left(\omega_{\perp} / \omega_{z}=0.1\right)$. In all panels $N c_{0}=50 \hbar \omega_{\perp} l_{\perp}^{3}$ and $N c_{2}=-10 \hbar \omega_{\perp} l_{\perp}^{3}$. (a) Stability of a coreless vortex in the presence of linear Zeeman splitting. The vortex state becomes unstable for $g_{1}|\mathbf{B}| \gtrsim 0.2 \hbar \omega_{\perp}$. (b) The singular vortex remains stable despite linear Zeeman splitting. (c) Stability of the coreless vortex in the presence of quadratic Zeeman splitting. A stable region is found at all investigated values of $g_{2}|\mathbf{B}|$. (d) Stability of the singular vortex in the presence of quadratic Zeeman splitting. The vortex becomes unstable for a relatively small negative $g_{2}|\mathbf{B}|$. (Symbols as in Fig. 3.) 
[Figs. 10(c) and 10(d)]. For a sufficiently large positive quadratic Zeeman splitting, the ideal spinor basis to analyze the singular vortex core is oriented perpendicular to the magnetic field.

\section{STABILITY AND CORE STRUCTURE OF A POLAR VORTEX}

We now turn our attention to the polar phase of a spin1 BEC. In this case the interaction term $c_{2}>0$ in the Hamiltonian (2), and it is energetically favorable to minimize the spin magnitude everywhere in space so that $|\langle\hat{\mathbf{F}}\rangle|=0$. We take a representative spinor $\zeta=(0,1,0)^{T}$ whose macroscopic condensate spin quantization axis is oriented along the $z$ axis. The general spinor wave function may then be constructed from the macroscopic condensate phase $\phi$ and the spin rotations defined by the Euler angles $(\alpha, \beta, \gamma)$ as

$$
\zeta^{\mathrm{p}}=e^{i \phi} U(\alpha, \beta, \gamma)\left(\begin{array}{l}
0 \\
1 \\
0
\end{array}\right)=\frac{e^{i \phi}}{\sqrt{2}}\left(\begin{array}{c}
-e^{-i \alpha} \sin \beta \\
\sqrt{2} \cos \beta \\
e^{i \alpha} \sin \beta
\end{array}\right)
$$

It is beneficial to introduce the unit vector $\hat{\mathbf{d}}=$ ( $\cos \alpha \sin \beta, \sin \alpha \sin \beta, \cos \beta$ ) that defines the local direction of the condensate spin quantization. We may then write the spinor wave function in terms of $\hat{\mathbf{d}}$ as [23]

$$
\zeta^{\mathrm{p}}=\frac{e^{i \phi}}{\sqrt{2}}\left(\begin{array}{c}
-d_{x}+i d_{y} \\
\sqrt{2} d_{z} \\
d_{x}+i d_{y}
\end{array}\right)
$$

The unit vector $\hat{\mathbf{d}}$ takes values on a sphere and the condensate phase $\phi$ on a unit circle. The state of the spinor wave function, however, remains unchanged when a $\pi$ rotation of $\phi$ is combined with inverting the $\hat{\mathbf{d}}$ vector, so that the states $\zeta^{\mathrm{p}}(\phi, \hat{\mathbf{d}})=\zeta^{\mathrm{p}}(\phi+\pi,-\hat{\mathbf{d}})$ are identical. These states must be identified to avoid double counting, and the order-parameter space is therefore $\left[\mathrm{U}(1) \times S^{2}\right] / \mathbb{Z}_{2}$, from the condensate phase and rotations of $\hat{\mathbf{d}}$, factorized by the discrete two-element group $\mathbb{Z}_{2}$ due to the identification. The vector $\hat{\mathbf{d}}$ is thus taken to be unoriented and defines a nematic axis [24].

A singly quantized vortex in the polar phase can be formed as a $2 \pi$ winding of the condensate phase $\phi$ around a closed loop encircling the vortex core. Choosing the vortex line along the $z$ axis, we obtain

$$
\zeta^{1}=\frac{e^{i \varphi}}{\sqrt{2}}\left(\begin{array}{c}
-e^{-i \alpha} \sin \beta \\
\sqrt{2} \cos \beta \\
e^{i \alpha} \sin \beta
\end{array}\right)
$$

However, the nematic order also allows the formation of a vortex carrying half a quantum of circulation [11] (in analogy to the half-quantum vortices in superfluid liquid ${ }^{3} \mathrm{He}$ [4]), constructed as a $\pi$ winding of the macroscopic condensate phase together with a $\hat{\mathbf{d}} \rightarrow-\hat{\mathbf{d}}$ rotation of the nematic axis around a closed loop encircling the vortex core. For example, we may have

$$
\zeta^{1 / 2}=\frac{e^{i \varphi / 2}}{\sqrt{2}}\left(\begin{array}{c}
-e^{-i \varphi / 2} \\
0 \\
e^{i \varphi / 2}
\end{array}\right)=\frac{1}{\sqrt{2}}\left(\begin{array}{c}
-1 \\
0 \\
e^{i \varphi}
\end{array}\right) .
$$

(a)

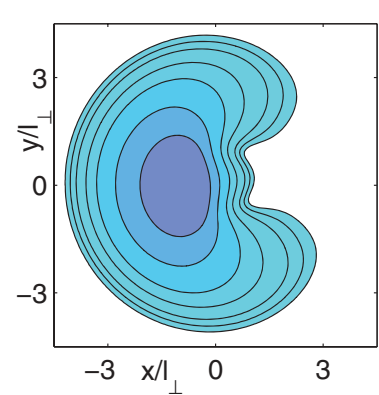

(c)

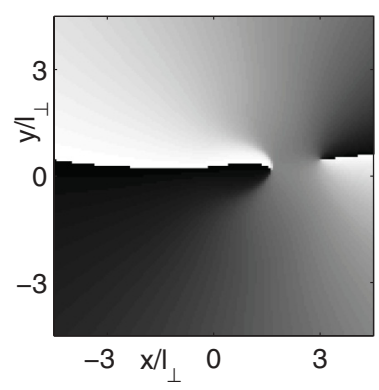

(b)

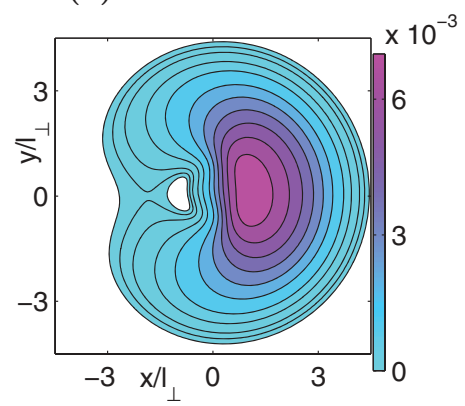

(d)

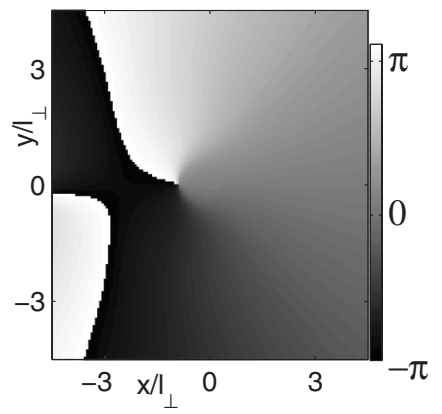

FIG. 11. (Color online) Stable core structure of the singular vortex in the polar phase shown in the $x y$ plane. (a),(b) Densities in $\zeta_{+}^{(z)}$ and $\zeta_{0}^{(z)}$, respectively. (c),(d) The corresponding phases. $\left(\zeta_{-}^{(z)}\right.$ is identical to $\zeta_{+}^{(z)}$ up to a global $\pi$ phase shift.) The spinor wave function exhibits vortex lines with highly deformed anisotropic cores in the spinor components.

Circulation is thus quantized in units of $\pi$, half the circulation of Eq. (11). This will be a crucial observation when we now analyze possible deformations of the core of a singly quantized vortex as energy is minimized.

In order to investigate the energetic stability of a singly quantized singular vortex in the polar phase of a spin-1 BEC, we numerically minimize the energy of the system in a rotating frame. We follow the same procedure as in the FM case and this time take a singular polar vortex of Eq. (11) with $\beta=$ $\pi / 4$ and $\alpha=0$ as an initial state of the numerical relaxation. As in the FM case of Eq. (7), the initial state is formed by overlapping vortex lines in all three spinor components. Upon minimizing the energy, the vortex cores of the individual spinor components separate. However, compared with the FM case, the splitting is now more complicated, as shown in Fig. 11. The result is highly deformed anisotropic vortex cores in the spinor components. The vortices in $\zeta_{+}$and $\zeta_{-}$overlap, but the one in $\zeta_{0}$ is displaced from the other two. There are no simultaneous density minima in all three spinor components, and the density is therefore nonzero everywhere. Similar splitcore solutions found by numerical calculation in a rotating 2D system [10] have resulted in some controversy regarding the number of vortices in the individual spinor components in the final configuration [16]. In the previous 2D studies the stable core structures were not classified. Here we show now how the split core in Fig. 11 can be identified as a topologypreserving splitting of the singly quantized vortex into a pair of half-quantum vortices as illustrated schematically in Fig. 1. 

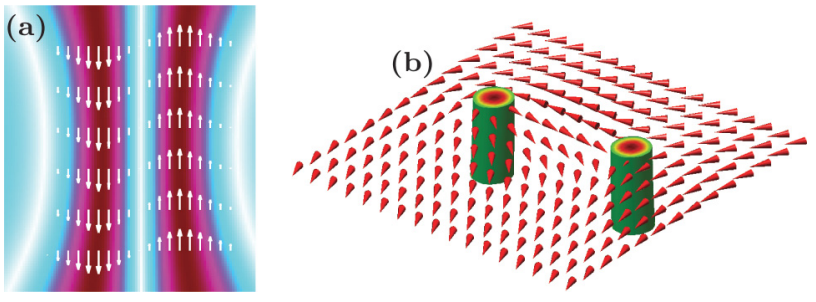

FIG. 12. (Color online) Splitting of the singly quantized vortex into two half-quantum vortices. (a) Spin magnitude $|\langle\hat{\mathbf{F}}\rangle|$ [color map from white $(|\langle\hat{\mathbf{F}}\rangle|=0)$ to red (dark gray) $(|\langle\hat{\mathbf{F}}\rangle|=1)]$ together with the spin vector (arrows), showing the FM cores with nonvanishing density. The spins are antiparallel in the two cores. (b) Nematic axis $\hat{\mathbf{d}}(r)$ together with the vortex cores [indicated by green (light gray) isosurfaces of $|\langle\hat{\mathbf{F}}\rangle|$, with increasing spin magnitude indicated by the color gradient inside]. Away from the vortex cores the topology of the initial singly quantized vortex is preserved. In the core region, $\hat{\mathbf{d}}$ winds by $\pi$ about each half-quantum vortex core. For visualization purposes, the unoriented $\hat{\mathbf{d}}$ field is shown as cones. Here a quadratic Zeeman shift has been introduced to ensure that $\hat{\mathbf{d}}$ lies in the $x y$ plane and the spins align with the $z$ axis.

In the numerical simulations the initial state of a singly quantized singular vortex in Eq. (11) is composed of three perfectly overlapping vortex lines in each of the three spinor components. The polar vortex consequently has a vanishing density at the line singularity of the polar order parameter of the spin-1 BEC. The singular vortex with zero density is energetically unstable with respect to core deformation. As the vortices of the individual spinor components move apart during energy relaxation, the density becomes nonvanishing everywhere in the vortex-core region. Similarly to the FM vortex case, we must therefore require that the spinor wave function becomes orthogonal to the ground-state manifold at the vortex singularity. This indicates that we must have $|\langle\hat{\mathbf{F}}\rangle|=$ 1 on the vortex line. We show in Fig. 12 the numerically calculated vortex core structure of a stable vortex whose initial state is the singular singly quantized vortex of Eq. (11). The displayed spin magnitude exhibits two clearly separated cores in which the peak value increases to $|\langle\hat{\mathbf{F}}\rangle|=1$, indicating the emergence of a FM core region for the vortex.

The formation of the FM cores can be understood from the same argument used to understand the polar core of the singular FM vortex in Sec. III and is illustrated in Fig. 1: The singular polar vortex (11), which is used as an initial state in the energy relaxation, implies a density-depleted core whose size is determined by $\xi_{n}$. However, accommodating a singularity of the polar order parameter by requiring $|\langle\hat{\mathbf{F}}\rangle|=1$ at the vortex line means that the length scale, and thus the associated bending energy, is determined by $\xi_{F}$. The energy of Eq. (11) can thus be lowered by having a nonvanishing atom density and by extending the core size from $\xi_{n}$ to $\xi_{F}$. In the case of a polar vortex this is achieved by spontaneously breaking the axial symmetry and forming two FM cores by the mechanism sketched in Fig. 1. The separation between the cores is of the order of $\xi_{F}$, depending also on the angular momentum of the system when it adjusts to the rotation frequency and on the density gradient due to the trap.

We may analyze this symmetry breaking of the vortex core by means of a basis transformation. We write the spinor in the (a)

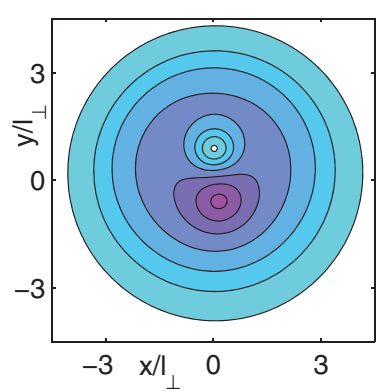

(c)

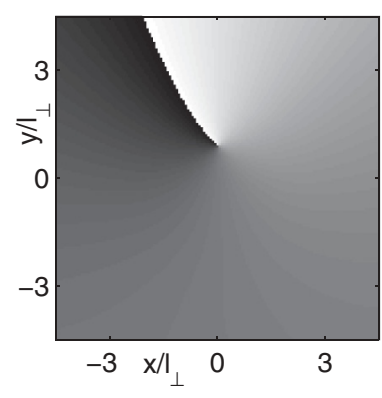

(b)

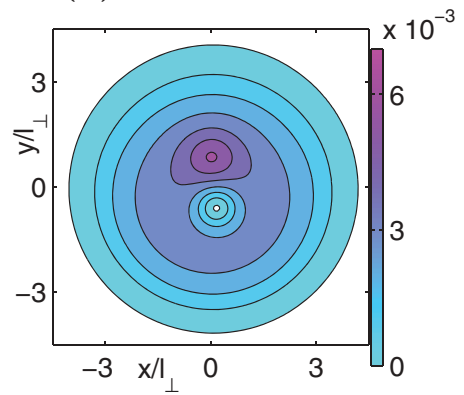

(d)

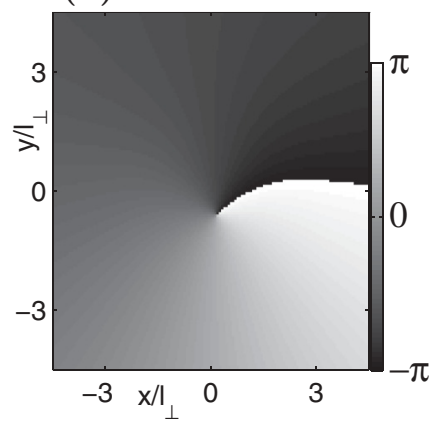

FIG. 13. (Color online) Spinor wave function of the stable singular vortex state from Fig. 11 after spinor-basis transformation such that spin is quantized along the $y$ axis. (a),(b) Densities in $\zeta_{+}^{(y)}$ and $\zeta_{-}^{(y)}$, respectively. (c),(d) The corresponding phases. The component $\zeta_{0}^{(y)}$ (not shown) is unpopulated. The previously complex structure can now be identified as a pair of half-quantum vortices.

basis of spin projection onto the axis given by the spin vector in the FM core. For the case of Fig. 11(a), the spins in the two cores align (antialign) with the $y$ axis and we calculate $\zeta^{(y)}=U^{-1}(\pi / 2, \pi / 2,0) \zeta^{(z)}$. The resulting spinor then shows displaced vortex lines in $\zeta_{ \pm}^{(y)}$ while the density vanishes in $\zeta_{0}^{(y)}$ (Fig. 13). In the new spinor basis, the vortex lines in $\zeta_{ \pm}^{(y)}$ coincide precisely with the spin maxima, as shown in Fig. 14.

We can now identify the core structure emerging from the splitting of the singular vortex by comparing the spin-rotated state $\zeta^{(y)}$ with Eq. (12). We then find that each vortex line in $\zeta^{(y)}$ has exactly the form of a half-quantum vortex. The split-core configuration may thus be interpreted as a splitting
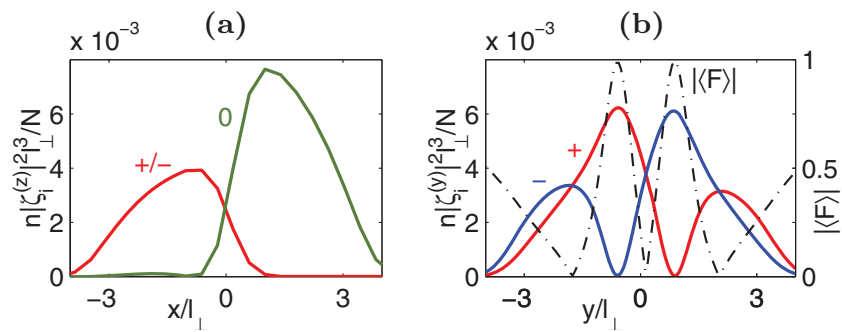

FIG. 14. (Color online) (a) Density profiles of the spinor components on the axis connecting their density minima (cf. Fig. 11). Lines are labeled with the spinor-component index. Note that $\left|\zeta_{ \pm}\right|$ exactly overlap. (b) Spinor-component density profiles in $\zeta^{(y)}$ after basis transformation (cf. Fig. 11) plotted along the axis connecting the half-quantum vortices. The spin magnitude $|\langle\hat{\mathbf{F}}\rangle|$ (black dash-dotted line) shows the FM cores. Unpopulated $\zeta_{0}^{(y)}$ is not shown. 
of the singly quantized vortex into a pair of half-quantum vortices with FM cores. The topological charges of vortices are here additive and topology is therefore preserved when the singly quantized vortex splits into the pair of half-quantum vortices. This can also be inferred from the behavior of the nematic axis $\hat{\mathbf{d}}$. Figure 12(b) shows $\hat{\mathbf{d}}$ in a numerical solution together with the FM cores of the half-quantum vortices. Away from the vortices, there is no net winding in $\hat{\mathbf{d}}$ on a path enclosing the vortices. However, on a path that encircles only one vortex core, $\hat{\mathbf{d}}$ turns by $\pi$, indicating the emergence of a disclination plane as indicated in Fig. 1.

As in the case of a FM vortex, the core deformation can be explained in terms of the vortex topology and the energetic hierarchy of different length scales (see Fig. 1). Outside the vortex-core region of size $\xi_{F}$, where the order-parameter bending energy is not sufficient to excite the system away from the polar ground-state manifold, we have $|\langle\hat{\mathbf{F}}\rangle|=0$ and the topological properties of the initial singly quantized singular vortex are preserved. This is indicated by the unit winding of the macroscopic condensate phase around any closed loop encircling the entire vortex core and by the nematic vector field outside the core region. It is only inside the core of size $\xi_{F}$ that the strong order-parameter bending energy restores the symmetry of the full spin-1 condensate wave function by exciting the system out of the polar ground-state manifold and by allowing the complete range of spin values $|\langle\hat{\mathbf{F}}\rangle|$ from 0 to 1 . The local deformation of the core is topologically possible due to the nematic order of the polar phase, where the axis $\hat{\mathbf{d}}$ is unoriented, with the opposite orientations $\hat{\mathbf{d}}=-\hat{\mathbf{d}}$ identified. The core deformation mechanism of the vortex line is related to the point-defect deformation into a singular ring where the nematic order allows the spontaneous breaking of the spherical defect-core symmetry [23]. In the $B$ phase of superfluid liquid ${ }^{3} \mathrm{He}$, a stable nonaxisymmetric singular vortex with a nonzero superfluid density at the core was theoretically predicted in Refs. [57,58] and experimentally observed in Ref. [59]. The ${ }^{3} \mathrm{He} A$-phase core was explained to consist of two half-quantum vortices. In the high-pressure regime the axial symmetry of the vortex is restored but the core can still remain in the $A$ phase with a nonvanishing superfluid density [70].

We find that the singular vortex splits into a pair of half-quantum vortices by the mechanism described above for all investigated parameter regimes. However, we find a critical rotation frequency of $\Omega \simeq 0.3 \omega_{\perp}$ below which the vortices start exiting the atom cloud. Figure 15 shows the energetic stability of the half-quantum vortex pair obtained from splitting of a singly quantized vortex in both isotropic and oblate $\left(\omega_{\perp} / \omega_{z}=0.1\right)$ traps. The energetic ground state of a rotating polar spin-1 BEC can consist of half-quantum vortices $[26,28]$, and increasing the rotation frequency leads to nucleation of more half-quantum vortices in addition to the split core of the initial singular vortex.

The splitting mechanism of the singly quantized vortex is qualitatively similar also when a weak Zeeman splitting due to a magnetic field is introduced. However, as shown in Fig. 15(c), a linear Zeeman splitting of $g_{1} B \gtrsim 0.4 \hbar \omega_{\perp}$ causes the resulting pair of half-quantum vortices to become energetically unstable at all rotational frequencies. By contrast, the vortex pair remains stable above $\Omega \simeq 0.3 \omega_{\perp}$ for the entire (a)

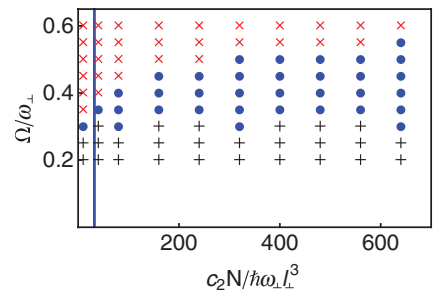

(c)

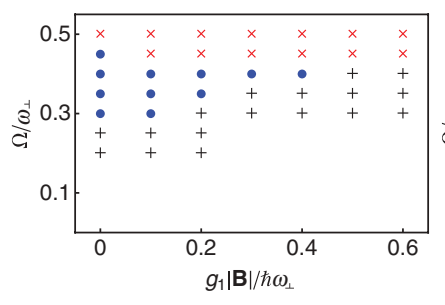

(b)

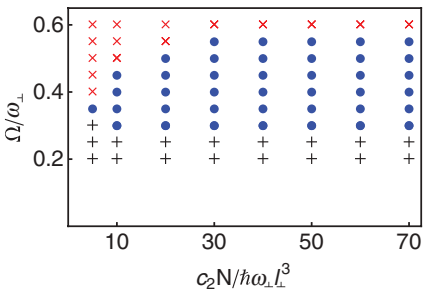

(d)

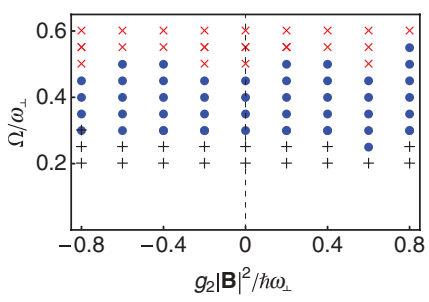

FIG. 15. (Color online) Energetic stability of the split-core singular vortex in the polar phase. (a) Stability in the isotropic trap for varying $c_{2}$ using $N c_{0}=1000 \hbar \omega_{\perp} l_{\perp}^{3}$. The vertical line marks the value $c_{0} / c_{2} \simeq 31$ for ${ }^{23} \mathrm{Na}$ [71]. (b) Stability in the oblate trap $\left(\omega_{\perp} / \omega_{z}=0.1\right)$ for varying $c_{2}$ using $N c_{0}=50 \hbar \omega_{\perp} l_{\perp}^{3}$. (c),(d) Stability of the split-core singular vortex in the presence of linear and quadratic Zeeman splitting, respectively. Increasing linear Zeeman splitting renders the singular vortex unstable, whereas the stability is robust against quadratic Zeeman splitting. In the slowly rotating region for all panels, the instability of the split-core singular vortex may be either towards the vortex-free state, or towards the state with a single half-quantum vortex. (Symbols as in Fig. 3.)

range of quadratic Zeeman splittings considered $\left(-0.8 \hbar \omega_{\perp} \leqslant\right.$ $\left.g_{2}|B|^{2} \leqslant 0.8 \hbar \omega_{\perp}\right)$ [Fig. 15(d)].

\section{CONCLUSIONS}

We have demonstrated that a singular, singly quantized vortex can be energetically stable in the FM phase of a spin-1 BEC, despite the existence of a stable coreless vortex with lower energy (increasing the rotation frequency in a vortex-free BEC also leads to nucleation of coreless vortices). This implies that, even though singular vortices would not be nucleated by rotation alone, a singly quantized vortex created, for example, by phase imprinting would remain stable in the rotating system. This provides an interesting opportunity for controlled studies of a singular vortex line in a ground-state manifold with a broken $\mathrm{SO}(3)$ symmetry. Such a system supports only two topological classes of vortices: those that can be locally deformed to a vortex-free configuration and those that are topologically equivalent to a singly quantized singular vortex. Experimentally, one could phase-imprint overlapping vortex lines in each of the three spinor wave-function components. The resulting structure represents a singular spin-1 vortex with a vanishing density at the vortex line. The core of such a vortex then deforms to an energy-minimized configuration within the same topological equivalence class.

The stable vortex core in the FM phase is formed by nonoverlapping vortex lines in the three spinor components. We have demonstrated that this seemingly complex core structure can be understood in terms of a combination of the vortex topology and the energetics of characteristic length 
scales. By deforming the core of a singly quantized, singular vortex in the FM phase so as to maintain a nonzero density everywhere, instead accommodating the singularity by forcing $|\langle\hat{\mathbf{F}}\rangle|=0$, the gradient contribution to the energy is lowered. The reason is that the size of the defect core is then determined by the spin healing length $\xi_{F}$ which is in general larger than the characteristic size $\xi_{n}$ of a defect core where the density goes to zero. In other words, in the larger-core-size case with a nonvanishing atom density, the gradient energy restores the full symmetry of the spin-1 condensate wave function within the core region. The system then simultaneously exhibits two different order-parameter symmetries: maximal unbroken symmetry inside the core of size $\xi_{F}$ and a broken symmetry (of the FM phase) outside the vortex core.

The core deformation mechanism results in a singular vortex whose core is also filled with atoms in the polar phase. The spin vector winds by $2 \pi$ as the core is encircled. The single vortex core can be explicitly restored in the spinor by judicious choice of spinor basis.

In the polar phase, we have shown that a singly quantized vortex is stabilized by a spontaneous breaking of axial symmetry. The resulting stable defect is a pair of half-quantum vortices with FM cores, which is stable in a sufficiently rapidly rotating trap. The formation of the FM cores avoids depleting the density in the vortex core. This is energetically favorable by the same reasoning that was applied to explain the polar core of the singular vortex in the FM phase. The resulting spinor wave function is analyzed and the vortex structure identified through a rotation of the spinor basis, so that in the rotated basis the half-quantum vortices appear as separate vortex lines in the $\zeta_{ \pm}$components.

\section{ACKNOWLEDGMENTS}

We acknowledge financial support from the EPSRC and the Leverhulme Trust.

\section{APPENDIX: BASIS TRANSFORMATION FOR THE FM VORTEX}

In the numerical simulations we found that the singular FM vortex relaxes to a stable configuration formed by nonoverlapping vortex lines in the three spinor components, as shown in Fig. 5(a). By an appropriately chosen basis transformation we showed that this seemingly complex vortex structure can be identified as a single, singular vortex with the line singularity populated by atoms in the polar phase [Fig. 5(b)]. In this Appendix we demonstrate this basis transformation through a qualitative analytic treatment and show how the core structure of the singular FM vortex may be identified.

For simplicity, we implement the basis transformation by starting from the final configuration of the singular vortex with a single vortex line as in Figs. 5(b) and 7 and rotating to the configuration of nonoverlapping vortex lines in the three spinor components [see, e.g., Fig. 5(a)]. The analytic expressions become notably simpler in the case of the axisymmetric vortex of Fig. 7 than with the one displaying a more complex spin rotation in Figs. 4(b) and 5, but the basic principle of the transformation is the same in both cases. In order to describe the vortex of Fig. 7 we rewrite the singular vortex displaying a radial disgyration of the spin vector $\zeta^{\text {sv }}$ of Eq. (8) in the following form:

$$
\zeta=\frac{1}{\sqrt{2}}\left(\begin{array}{c}
\sqrt{2}(\cos \varphi-i \sin \varphi) f \cos ^{2} \frac{\beta}{2} \\
g \sin \beta \\
\sqrt{2}(\cos \varphi+i \sin \varphi) f \sin ^{2} \frac{\beta}{2}
\end{array}\right) .
$$

Here we have introduced the profile function $f(x, y)$ and the notation

$$
g \sin \beta=\sqrt{2-f^{2}\left(1+\cos ^{2} \beta\right)} .
$$

The profile function $f$ describes the mixing of the FM and the polar phases in the core region of the vortex. For $\beta \neq \pi$ we obtain a radial disgyration with nonvanishing density at the singularity (for $\beta=\pi$ and $f=1$ we recover a vortex with zero density at the singularity). We assume $f$ to be monotonically increasing from 0 at the $z$ axis (the vortex singularity), reaching 1 outside the vortex core of size $\xi_{F}$. For the numerically minimized stable solution of Fig. 7 the parameter $\beta$ is not constant; close to the vortex core we have $\beta \simeq \pi / 2$ and far away from the vortex $\beta \rightarrow \pi$. The magnitude of the spin vector may be evaluated from Eq. (A1), yielding $|\langle\hat{\mathbf{F}}\rangle|=f \sqrt{2-f^{2}}$. At the line singularity $|\langle\hat{\mathbf{F}}\rangle|=0$, representing the polar phase, and outside the core region $|\langle\hat{\mathbf{F}}\rangle|=1$, which corresponds to the FM phase. In between $|\langle\hat{\mathbf{F}}\rangle|$ continuously varies between these two values, indicating the mixing of the two phases.

When we perform the rotation of Eq. (A1) by the angle of $-\pi / 2$ with respect to the $x$ axis, we obtain the spinor wave function

$$
\zeta^{(-x)}=\frac{1}{2}\left(\begin{array}{c}
f(\cos \varphi-i \sin \varphi \cos \beta)+g \sin \beta \\
\sqrt{2} f(-\cos \varphi \cos \beta+i \sin \varphi) \\
f(\cos \varphi-i \sin \varphi \cos \beta)-g \sin \beta
\end{array}\right) .
$$

The spinor wave functions are of the form $\left(x-x_{0}\right)+i \eta(y-$ $\left.y_{0}\right)$, indicating that a singly quantized vortex line is located at $\left(x_{0}, y_{0}\right)$. The anisotropy of the vortex core is described by the parameter $\eta$. The singularity in $\zeta_{0}^{(-x)}$ therefore is on the $z$ axis, while those in $\zeta_{ \pm}^{(-x)}$ are displaced to $\left(\mp x_{0}, y_{0}=0\right)$. Here $x_{0}$ is determined as the point at which $f\left(x_{0}, 0\right)=$ $g\left(x_{0}, 0\right) \sin \beta$, resulting in $f\left(x_{0}, 0\right)=\sqrt{2 /\left(2+\cos ^{2} \beta\right)}$. The vortex configuration of Eq. (A3) with three spatially separated vortex lines is analogous to that shown in Fig. 5(a).
[1] D. R. Tilley and J. Tilley, Superfluidity and Superconductivity, 3rd ed. (IOP Publishing, Bristol, UK, 1990).

[2] C. Pethick and H. Smith, Bose-Einstein Condensation in Dilute Gases (Cambridge University Press, Cambridge, 2002).
[3] J. Stenger, S. Inouye, D. M. Stamper-Kurn, H. Miesner, A. P. Chikkatur, and W. Ketterle, Nature (London) 396, 345 (1998).

[4] D. Vollhardt and P. Wölfle, The Superfluid Phases of Helium 3 (Taylor \& Francis, London, 1990). 
[5] M. M. Salomaa and G. E. Volovik, Rev. Mod. Phys. 59, 533 (1987).

[6] G. E. Volovik, The Universe in a Helium Droplet (Oxford University Press, Oxford, 2003).

[7] A. Vilenkin and E. P. S. Shellard, Cosmic Strings and Other Topological Defects (Cambridge University Press, Cambridge, 1994).

[8] N. Manton and P. Sutcliffe, Topological Solitons (Cambridge University Press, Cambridge, 2004).

[9] T.-L. Ho, Phys. Rev. Lett. 81, 742 (1998).

[10] S.-K. Yip, Phys. Rev. Lett. 83, 4677 (1999).

[11] U. Leonhardt and G. Volovik, JETP Lett. 72, 46 (2000).

[12] J. Ruostekoski and J. R. Anglin, Phys. Rev. Lett. 86, 3934 (2001).

[13] U. Al Khawaja and H. Stoof, Nature (London) 411, 918 (2001).

[14] H. T. C. Stoof, E. Vliegen, and U. Al Khawaja, Phys. Rev. Lett. 87, 120407 (2001).

[15] T. Isoshima and K. Machida, Phys. Rev. A 66, 023602 (2002).

[16] T. Mizushima, K. Machida, and T. Kita, Phys. Rev. A 66, 053610 (2002).

[17] T. Mizushima, K. Machida, and T. Kita, Phys. Rev. Lett. 89, 030401 (2002).

[18] J.-P. Martikainen, A. Collin, and K.-A. Suominen, Phys. Rev. A 66, 053604 (2002).

[19] E. J. Mueller and T.-L. Ho, Phys. Rev. Lett. 88, 180403 (2002).

[20] K. Kasamatsu, M. Tsubota, and M. Ueda, Phys. Rev. Lett. 91, 150406 (2003).

[21] R. A. Battye, N. R. Cooper, and P. M. Sutcliffe, Phys. Rev. Lett. 88, 080401 (2002).

[22] C. M. Savage and J. Ruostekoski, Phys. Rev. Lett. 91, 010403 (2003).

[23] J. Ruostekoski and J. R. Anglin, Phys. Rev. Lett. 91, 190402 (2003).

[24] F. Zhou, Int. J. Mod. Phys. B 17, 2643 (2003).

[25] C. M. Savage and J. Ruostekoski, Phys. Rev. A 68, 043604 (2003).

[26] J. W. Reijnders, F. J. M. van Lankvelt, K. Schoutens, and N. Read, Phys. Rev. A 69, 023612 (2004).

[27] J. Ruostekoski, Phys. Rev. A 70, 041601 (2004).

[28] E. J. Mueller, Phys. Rev. A 69, 033606 (2004).

[29] H. Saito, Y. Kawaguchi, and M. Ueda, Phys. Rev. Lett. 96, 065302 (2006).

[30] L. Santos and T. Pfau, Phys. Rev. Lett. 96, 190404 (2006).

[31] G. W. Semenoff and F. Zhou, Phys. Rev. Lett. 98, 100401 (2007).

[32] R. Barnett, A. Turner, and E. Demler, Phys. Rev. A 76, 013605 (2007).

[33] V. Pietilä and M. Möttönen, Phys. Rev. Lett. 102, 080403 (2009).

[34] J. A. M. Huhtamäki, T. P. Simula, M. Kobayashi, and K. Machida, Phys. Rev. A 80, 051601 (2009).

[35] M. Kobayashi, Y. Kawaguchi, M. Nitta, and M. Ueda, Phys. Rev. Lett. 103, 115301 (2009).

[36] M. Takahashi, V. Pietilä, M. Möttönen, T. Mizushima, and K. Machida, Phys. Rev. A 79, 023618 (2009).

[37] T. P. Simula, J. A. M. Huhtamäki, M. Takahashi, T. Mizushima, and K. Machida, J. Phys. Soc. Jpn. 80, 013001 (2011).

[38] M. O. Borgh and J. Ruostekoski, Phys. Rev. Lett. 109, 015302 (2012).

[39] M. Nitta, K. Kasamatsu, M. Tsubota, and H. Takeuchi, Phys. Rev. A 85, 053639 (2012).
[40] T. Busch and J. R. Anglin, Phys. Rev. Lett. 87, 010401 (2001).

[41] P. Öhberg and L. Santos, Phys. Rev. Lett. 86, 2918 (2001).

[42] P. Kevrekidis, H. Nistazakis, D. Frantzeskakis, B. Malomed, and R. Carretero-González, Eur. Phys. J. D 28, 181 (2004).

[43] N. G. Berloff, Phys. Rev. Lett. 94, 120401 (2005).

[44] U. Shrestha, J. Javanainen, and J. Ruostekoski, Phys. Rev. Lett. 103, 190401 (2009).

[45] C. Yin, N. G. Berloff, V. M. Pérez-García, D. Novoa, A. V. Carpentier, and H. Michinel, Phys. Rev. A 83, 051605 (2011).

[46] H. E. Nistazakis, D. J. Frantzeskakis, P. G. Kevrekidis, B. A. Malomed, and R. Carretero-González, Phys. Rev. A 77, 033612 (2008).

[47] R. Carretero-González, D. J. Frantzeskakis, and P. G. Kevrekidis, Nonlinearity 21, R139 (2008).

[48] A. E. Leanhardt, Y. Shin, D. Kielpinski, D. E. Pritchard, and W. Ketterle, Phys. Rev. Lett. 90, 140403 (2003).

[49] L. S. Leslie, A. Hansen, K. C. Wright, B. M. Deutsch, and N. P. Bigelow, Phys. Rev. Lett. 103, 250401 (2009).

[50] J.-Y. Choi, W. J. Kwon, and Y.-i. Shin, Phys. Rev. Lett. 108, 035301 (2012).

[51] M. Vengalattore, S. R. Leslie, J. Guzman, and D. M. StamperKurn, Phys. Rev. Lett. 100, 170403 (2008).

[52] J. Kronjäger, C. Becker, P. Soltan-Panahi, K. Bongs, and K. Sengstock, Phys. Rev. Lett. 105, 090402 (2010).

[53] L. E. Sadler, J. M. Higbie, S. R. Leslie, M. Vengalattore, and D. M. Stamper-Kurn, Nature (London) 443, 312 (2006).

[54] P. W. Anderson and G. Toulouse, Phys. Rev. Lett. 38, 508 (1977).

[55] N. D. Mermin and T.-L. Ho, Phys. Rev. Lett. 36, 594 (1976).

[56] S. Kobayashi, Y. Kawaguchi, M. Nitta, and M. Ueda, arXiv:1204.0202v1 [Phys Rev. A (to be published)].

[57] E. V. Thuneberg, Phys. Rev. Lett. 56, 359 (1986).

[58] M. M. Salomaa and G. E. Volovik, Phys. Rev. Lett. 56, 363 (1986).

[59] Y. Kondo, J. S. Korhonen, M. Krusius, V. V. Dmitriev, Y. M. Mukharsky, E. B. Sonin, and G. E. Volovik, Phys. Rev. Lett. 67, 81 (1991).

[60] T. Ohmi and K. Machida, J. Phys. Soc. Jpn. 67, 1822 (1998).

[61] J. Lovegrove, M. O. Borgh, and J. Ruostekoski (unpublished).

[62] N. D. Mermin, Rev. Mod. Phys. 51, 591 (1979).

[63] In ${ }^{3} \mathrm{He}$ the angular momentum is fixed at the cylinder wall, fixing the boundary condition of the coreless-vortex texture. For a fixed boundary condition the system can exhibit a conserved winding number defined by an integral of the spin-vector expression similar to the topological charge density of a point defect over the surface covering the upper hemisphere [4].

[64] J. Javanainen and J. Ruostekoski, J. Phys. A 39, L179 (2006).

[65] E. G. M. van Kempen, S. J. J. M. F. Kokkelmans, D. J. Heinzen, and B. J. Verhaar, Phys. Rev. Lett. 88, 093201 (2002). 
[66] Ü. Parts, J. M. Karimäki, J. H. Koivuniemi, M. Krusius, V. M. H. Ruutu, E. V. Thuneberg, and G. E. Volovik, Phys. Rev. Lett. 75, 3320 (1995).

[67] M. R. Matthews, B. P. Anderson, P. C. Haljan, D. S. Hall, C. E. Wieman, and E. A. Cornell, Phys. Rev. Lett. 83, 2498 (1999).

[68] A. E. Leanhardt, A. Görlitz, A. P. Chikkatur, D. Kielpinski, Y. Shin, D. E. Pritchard, and W. Ketterle, Phys. Rev. Lett. 89, 190403 (2002).
[69] M. F. Andersen, C. Ryu, P. Clade, V. Natarajan, A. Vaziri, K. Helmerson, and W. D. Phillips, Phys. Rev. Lett. 97, 170406 (2006).

[70] M. M. Salomaa and G. E. Volovik, Phys. Rev. Lett. 51, 2040 (1983).

[71] A. Crubellier, O. Dulieu, F. Masnou-Seeuws, M. Elbs, H. Knöckel, and E. Tiemann, Eur. Phys. J. D 6, 211 (1999). 